\title{
Forgetting Factor Least Square Parameter Identification Based on Tool Servo Speed Tracking of the Milling Process
}

\author{
Ke Xu' ${ }^{1}$, Jie Yang ${ }^{1,2, *}$, Weiwei Fan ${ }^{1}$, Chuansheng Tang ${ }^{3}$ and Tao $\mathrm{Li}^{4}$ \\ ${ }^{1}$ Henan Institute of Technology, Xinxiang Henan 453000, China \\ ${ }^{2}$ Henan Key Equipment Engineering Research Center for New Energy Power Generation, Xinxiang Henan 453000, China \\ ${ }^{3}$ Nanyang Institute of Technology, Nanyang Henan 473004, China \\ ${ }^{4}$ Department of Informatics, University of Zurich, Zurich 8050, Switzerland
}

Received 15 May 2020; Accepted 30 July 2020

\begin{abstract}
The tool servo system of the computer numerically controlled (CNC) machine milling process is a complex nonlinear system composed of a servomechanism, a cutting process, and a detection device. In the actual machining process, accurately establishing a system model is difficult due to the coupling of parameters and nonlinear factors. Simultaneously, system parameters change with the working environment (e.g., resistance increases with an increase in temperature), leading to a decrease in the surface quality of the work piece. To improve the online identification accuracy of milling process model parameters and effectively increase the influence of parameter changes on system performance, a tool speed model prediction adaptive tracking method based on forgetting factor least square identification was proposed in this study. First, the model was discretized in accordance with the structure and characteristics of the tool servo feed system in the machining process, and the model parameters of the system were identified using the forgetting factor least square method. Second, a model predictive tracking method based on adaptive parameter estimation was designed on the basis of the discrete model of the system. Lastly, the effectiveness of the proposed method in model parameter identification and tool speed tracking was verified via numerical simulation. Results show that when uncertain factors, such as noise exist in the system, the least square identification method based on the forgetting factor can more quickly and accurately realize the model parameter identification of a tool servo feed system in the milling process than the stochastic gradient (SG) identification method. Moreover, identification accuracy is 30 times higher than that of the SG identification method. The model predictive tracking control method based on forgetting factor least square identification can quickly track tool speed without overshooting in $0.035 \mathrm{~s}$. By contrast, the traditional minimum variance predictive control is completely invalid in the actual stage. The proposed method exhibits high accuracy in tool speed tracking and strong robustness to changes in model parameters.
\end{abstract}

Keywords: Milling, Tool servo feed system, Model predictive control, Model identification

\section{Introduction}

Milling refers to fixing a blank and using a high-speed rotating milling cutter to cut out the required shape and characteristics, which is one of the most important processes in computer numerically controlled (CNC) machining. Milling is typically composed of multiple axis movements, and the tool servo feed motion is a single axis motion in the depth direction, which plays a decisive role in the surface quality. In the common milling process, the tool servo feed motion system is driven by a direct current (DC) motor through the coupled ball screw to drive the cutter into the tool holder for cutting. This system exhibits the characteristics of a simple structure, low cost, and easy maintenance, and it can basically meet the requirements for conventional CNC machining servo motion, which is widely applied to automobile parts, coal mining machinery, petroleum machinery, construction machinery, and other fields [1-3].

With the increase of processing complexity and working environment, the requirements for the servo feed system of $\mathrm{CNC}$ machine tools have become increasingly higher. The

*E-mail address: yangti56@163.com

ISSN: 1791-2377 @ 2020 School of Science, IHU. All rights reserved.

doi:10.25103/iestr.134.02 drive formation of this system is developing toward the direction of high speed, high acceleration, and high precision. The tool servo system composed of a DC motor and a ball screw has a simple structure and low cost, which is the most commonly applied transmission mode of existing $\mathrm{CNC}$ machine tools. However, the torque signal is transformed into the tool motion signal by the power system of the DC motor. Consequently, multiple signals are designed during the transmission process, the system exhibits the phenomena of multiparameter coupling and magnetic circuit saturation, and it is difficult to establish an accurate model of the system. Simultaneously, the system model parameters will change with changes in the working environment (e.g., temperature and humidity), and thus, controlling the system is a challenging task.

Considering the aforementioned issues, scholars have conducted numerous studies on the influences of model parameter uncertainty and external disturbance on system performance [4-7]. However, their methods are based on a deterministic model of a system. An accurate mathematical model of the tool servo feed system is difficult to establish in a complex and changeable environment. Therefore, determining how to realize the online identification of model parameters and the high-precision control of servo feed motion in the milling process on the basis of changes in 
model parameters has important practical significance for improving the machining accuracy of parts.

The forgetting factor least square identification method is adopted to identify the model parameters of a discrete tool servo feed system. A model adaptive tool speed tracking method is also designed to solve the problem of highprecision tracking of the milling process tool servo feed system under the condition of uncertain parameters. Thus, the proposed methods provide a scheme for the design and development of a high-performance servo system of CNC machine tools.

\section{State of the art}

To address the difficulty in accurately establishing the milling process model, most existing studies have focused on the motor power source in a machining servo system [823]. Odhano et al. [8] provided theoretical guidance for different motor identification methods, but did not present a specific identification process. $\mathrm{Xu}$ et al. [9] proposed a stochastic gradient (SG) model parameter identification method based on the forgetting factor for the regression model of a permanent magnet synchronous motor. The proposed method involved a considerable amount of calculation, and its convergence speed and accuracy were limited. To improve the estimation accuracy of a motor system, Qu et al. [10] developed a method for estimating the energy consumption parameters of cluster motors. Only the current information was used in the estimation process, and the dynamic estimation capability should be improved. Accetta et al. [11] applied a genetic algorithm to realize the optimal estimation of a linear motor. However, the size of the initial population was difficult to determine. If the scale of the proposed method was too small, then accuracy was low; if too many rules existed, then convergence was poor. Fagiano et al. [12] used the circuit breakers of industrial sensors to estimate motor parameters, but the introduction of circuit breakers reduced the reliability of the system. To improve the convergence speed and estimation accuracy of identification, Zhang et al. [13] introduced an adaptive linear neural network into the parameter identification of a permanent magnet synchronous motor. The structure and node number of the neural network resulted in design difficulties and were frequently difficult to determine. Scholars had conducted considerable study on machining process control [14-26]. Chatter was an unstable self-excited vibration phenomenon that leaded to tool wear, poor surface finish, and downhill milling operation. To improve the machining quality of the parts of the machining process, Paul et al. [14] presented an active flutter suppression strategy that combined proportional-derivative/proportionalintegral-derivative (PID) control with type 2 fuzzy logic control, and performed system stability analysis and experimental verification on the basis of Lyapunov stability theory. Although the proposed method improved the robustness of the system to external disturbances and model parameter changes, the controller used error and error rate as the control input and control gain as the output, making the structure complex and reducing dynamic response capability. Angel et al. [15] proposed a fractional-order PID controller that increased the self-use of the controller design and the capability to suppress system parameter perturbation. However, its effect was not good when a parameter suddenly changed. To improve the trajectory tracking capability of a controller, Corapsiz et al. [16] studied the performance of three PID control methods, PID based on the observer and feedforward compensation torque control for a three-axis $\mathrm{CNC}$ manipulator. The three aforementioned methods based on the system model were sensitive to changes in parameters. To minimize the contour error of 2-axis and 3-axis CNC machine tools, Hanifzadegan et al. [17] adopted a design method of multiple-input and multiple-output linear parameter change feedback controller. The proposed method applied a controller design technique based on linear matrix equality to the linear time-invariant $\mathrm{CNC}$ machine tool model. Simulation and experiments in the frequency and time domains showed that the proposed control method exhibited higher potential for reducing roaming errors than the traditional contour control method. However, the proposed control method was strongly limited in parameter variation and suffered from difficulty in meeting the high-precision requirement. To solve the optimal process planning problem of minimum turning time, Heydari et al. [18] adopted an optimal time control method when various equipment and process constraints are present. Zhou and Wu et al. [19-20] applied a modern robust control to the cutting process to improve the robustness of a system to parameter perturbation. Their simulation and experimental results showed that although the proposed method was robust to system parameter perturbations, its design was based on the maximum robust boundary, which required high control cost and affected the dynamic performance of a system. To reduce the influences of uncertainties, such as modeling errors, parameter changes, friction, linear and nonlinear measurement errors on the motion accuracy of a system, Huang et al. [21] successfully applied a state-space disturbance observer to the parameter variation of the servo motor's speed and current loops and to the uncertainty estimation and compensation of the current measurement problem, and then proposed a self-tuning method. Predictive control was a modern robust control method that used the information of the previous time to predict and estimate the current time. Cairano et al. [22] applied the proposed method to the motion control of a machine tool's machining process. The proposed method optimized the product quality and flexibility of the manufacturing process by coordinating the actuator and developing the operating range of the entire actuato, satisfied the actuator range and dynamic constraints, and ensured correct processing. Finite time processing for a given spatial pattern was implemented in real time even when computing resources were limited. However, the proposed method was sensitive to changes in model parameters and unsuitable for high-precision servo motion. Yuan et al. [23] combined Bayesian estimation with model predictive control to estimate model parameters. However, the control performance of the method was highly dependent on estimation accuracy. Moreover, Bayesian estimation was relatively complex and was not used in industrial applications.

On the basis of the preceding studies, a tool servo feed rate tracking method based on forgetting factor least square identification was proposed for the milling process. Forgetting factor least square identification was employed to identify the model parameters of a tool servo feed system, and an adaptive model predictive controller was designed to realize the precise tracking of the tool servo feed system in an uncertain milling process to improve the robustness and tracking capability of the system.

The remainder of this study is organized as follows. Section 3 describes the mechanical structure of the tool servo feed system in the milling process, constructs a 
dynamic model of the system, identifies the nominal parameters of the system model on the basis of forgetting factor least square identification, and designs a model predictive speed controller based on the adaptive identification of model parameters. Section 4 compares the proposed method with the SG identification method and the traditional minimum variance predictive control method by using MATLAB numerical simulation, and then verifies the validity and superiority of the proposed method. Lastly, Section 5 provides the conclusions.

\section{Methodology}

\subsection{Mathematical model of tool feed servo system used in} the milling process

Fig. 1 shows the structure diagram of the short arc milling process. The DC motor drives a ball screw through the coupling to drive the cutter on the cutter holder for cutting. In the model, $M$ denotes the DC motor, $J_{n}$ indicates the moment of inertia of the DC motor, $r_{0}$ represents the internal resistance of the DC motor, DC denotes the DC power supply, and $I$ indicates the equivalent load rotation inertia for the system that acts on the rotating shaft of the DC servo motor. $\theta_{m}$ denotes the rotation angle of the transmission mechanism, $L$ indicates the length of the ball screw guide rail, and $k$ represents the transmission stiffness. $F$ denotes the transmission force received by the executive part, and its driving force is exerted by the ball screw. $F_{0}$ indicates the external load, $m$ represents the quality of the executive part, and $c_{r}$ denotes the viscous friction coefficient of the guide rail. $x$ and $x_{0}$ represent the input and output displacements, respectively.

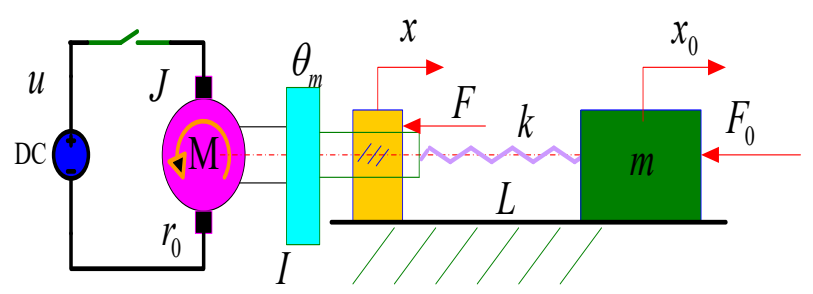

Fig. 1. Structure diagram of the short arc milling process

The servo motion model of the milling process can be expressed as follows:

$\left\{\begin{array}{l}F=m \ddot{x}_{0}+c_{r} \dot{x}_{0}+F_{0}=k\left(x-x_{0}\right) \\ T_{m}=J_{n} \dot{\omega}+B_{n} \omega+T_{L}=k_{T} I_{a} \\ U_{a}=I_{a} R_{a}+k_{d} \omega+L_{a} \dot{I}_{a}\end{array}\right.$

where $T_{m}, T_{L}, B_{n}$, and $k_{T}$ respectively represent the electromagnetic torque, load torque, viscous friction coefficient, and electromagnetic torque coefficient of the DC motor. $U_{a}, I_{a}, R_{a}, L_{a}$, and $k_{d}$ respectively denote the input voltage, input resistance current, resistance, inductance, and potential coefficient of the DC motor. $\theta$ indicates the position of the rotor, and $\dot{\theta}=\omega$ is the angular velocity of the rotor.
Disregarding the influences of the external load and the load torque, Eq. (1) can obtain the transmission function of the system tool speed to input voltage. Its transmission function can be expressed as follows:

$G(s)=\frac{\Omega(s)}{U(s)}=\frac{k_{M}}{\left(T_{M} S+1\right)\left(T_{E} S+1\right)}$

where $k_{M}=\frac{k_{T}}{R_{a} B_{n}+k_{d} k_{T}}, T_{M}=\frac{R_{a} J_{n}}{R_{a} B_{n}+k_{d} k}$, and $T_{E}=\frac{L_{a}}{R_{a}}$ represent the gain coefficient, mechanical time constant, and electrical time constant of the servo system in the milling process. In this model, motor resistance $R_{a}$, viscous friction coefficient $B_{n}$, moment of inertia $J_{n}$, and motor inductance $L_{a}$ will change with a change in the working environment of the servo system during the milling process, forming an uncertain milling servo system. The least square method exhibits the advantages of a simple principle, fast convergence, and easy understanding and programming; it is a highly effective estimation method for system parameters. Section 3.2 discusses the use of the forgetting factor recursive least square method in the online identification of system model parameters.

\subsection{Servo system model parameter forgetting factor least} square identification during the milling process

The least square method exhibits the advantages of a simple principle and fast convergence; it is also easy to understand and has been programmed. This method is highly effective for estimating system parameters. The following equation presents the online identification of system model parameters by using the forgetting factor least square method.

The following controlled auto-regressive model is considered as follows:

$A\left(z^{-1}\right) y(k)=B\left(z^{-1}\right) u(k-d)+\xi(k)$

where $\xi(k)$ indicates white noise, and the structural parameters $n_{a}, n_{b}$, and $d$ are known. Thus, the following equations can be obtained as follows:

$\left\{\begin{array}{l}A\left(z^{-1}\right)=1+a_{1} z^{-1}+a_{2} z^{-2}+\cdots+a_{n_{a}} z^{-n_{a}} \\ B\left(z^{-1}\right)=b_{0}+b_{1} z^{-1}+b_{2} z^{-2}+\cdots+b_{n_{a}} z^{-n_{b}}\end{array}\right.$

The purpose of parameter estimation is to determine $n_{a}+n_{b}+1$ parameters in accordance with measurable input and output. Eq. (3) can be written in the form of least squares as follows:

$$
\begin{aligned}
y(k)= & -a_{1} y(k-1)-\cdots-a_{n_{a}} y\left(k-n_{a}\right)+b_{0} u(k-d) \\
& +\cdots+b_{n_{b}} u\left(k-d-n_{b}\right)+\xi(k) \\
= & \psi^{T}(k) \theta(k)+\xi(k)
\end{aligned}
$$

According to the existing $L$ group input/output observation data, the least square estimation of the system parameters satisfying the specified performance index is obtained by batch processing method as follows: 
$\hat{\theta}=\left[\psi^{T} \psi\right]^{-1} \psi^{T} Y$

where

$$
Y=[y(1) \quad y(2) \cdots y(L)]^{T} \in R^{L \times 1}
$$

and $\psi=[\psi(1) \quad \psi(2) \cdots \psi(L)]^{T} \in R^{L \times\left(n_{a}+n_{b}+1\right)}$.

When Eq. (6) is applied to parameter estimation, the amount of data processed per time is considerable, consuming a large amount of memory but still cannot be used for online parameter estimation. In an adaptive control system, the controlled object can always provide new input/output data, and new information is expected to be used in improving estimation accuracy to realize online realtime estimation of plant parameters. The least square solution is changed to a recursive form; that is, the recursive least square parameter estimation algorithm can realize online estimation, and the estimation formula can be expressed as follows:

$$
\left\{\begin{array}{l}
\hat{\theta}(k)=\hat{\theta}(k-1)+K(k)\left[\mathrm{y}(\mathrm{k})-\psi^{T}(k) \hat{\psi}(k-1)\right] \\
K(k)=\frac{P(k-1) \psi(k)}{1+\psi^{T}(k) P(k-1) \psi(k)} \\
P(k)=\left[I-K(k) \psi^{T}(k)\right] P(k-1)
\end{array}\right.
$$

The object of study is an uncertain milling servo system; thus, the system parameters satisfy parameter mutation, although not frequently, or the parameters change slowly.

Meanwhile, the parameter mutation problem can be solved by resetting matrix $P$. In recursive least squares, the matrix is periodically reset to a sufficiently large number.

To address the problem of slow time-varying parameters, the so-called data saturation phenomenon with an increase in data will occur in the recursive least square method; that is, the sum becomes increasingly smaller with an increase in $k$, and thus, the correction capability becomes increasingly weaker, causing the newly collected input/output data to exert minimal effect on updating the parameter estimation value. When system parameters change, the recursive least square method cannot track the changes, resulting in the failure of real-time parameter estimation.

Therefore, the forgetting factor is introduced to improve the performance of the recursive least square method when the dynamic parameters of the milling process are changed. The estimation formula for the recursive least square method with a forgetting factor can be expressed as follows:

$$
\left\{\begin{array}{l}
\hat{\theta}(k)=\hat{\theta}(k-1)+K(k)\left[\mathrm{y}(\mathrm{k})-\psi^{T}(k) \hat{\psi}(k-1)\right] \\
K(k)=\frac{P(k-1) \psi(k)}{\lambda+\psi^{T}(k) P(k-1) \psi(k)} \\
P(k)=\frac{1}{\lambda}\left[I-K(k) \psi^{T}(k)\right] P(k-1)
\end{array}\right.
$$

where $\lambda$ is the forgetting factor. Thus, the following equations can be obtained as follows:

$$
\left\{\begin{array}{l}
\hat{\psi}(k)=\left[-y(k-1), \cdots,-y\left(k-n_{a}\right), u(k-d),\right. \\
\left.\quad \cdots, u\left(k-n_{a}-d\right), \xi(k-1), \cdots, \xi\left(k-n_{c}\right)\right]^{T} \\
\hat{\theta}=\left[\hat{a}_{1}, \hat{a}_{n_{a}}, \hat{b}_{1}, \hat{b}_{n_{b}}\right]^{T} \\
\hat{\xi}(k)=y(k)-\hat{y}(k)=y(k)-\hat{\psi}^{T}(k) \hat{\theta}
\end{array}\right.
$$

The performance index function is obtained as follows:

$$
J=\sum_{k=1}^{L} \lambda^{L-k}\left[y(k)-\psi^{T}(k) \hat{\theta}\right]^{2}
$$

The flowchart of the process identification of the cutting process parameters by using forgetting factor least square identification is illustrated in Fig. 2.

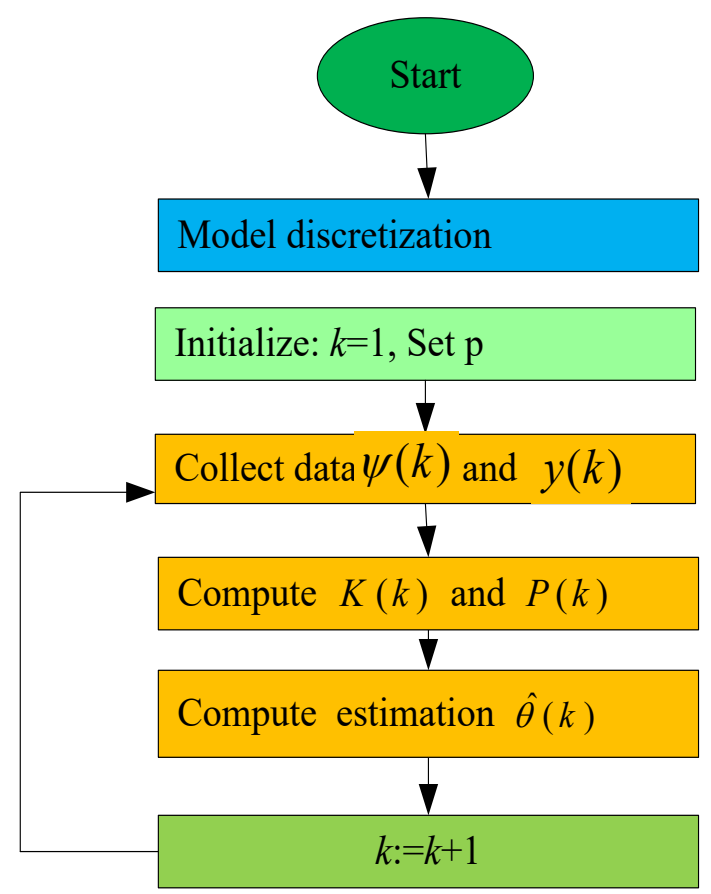

Fig. 2. Flowchart of parameter identification in the milling process

By using MATLAB, the identified model parameters can be transformed into the model parameters of the continuous system in Eq. (2) to determine model parameters $J_{n}$ and $B_{n}$ of the milling process.

\subsection{Design of model predictive tracker}

The reference trajectory is set as follows:

$$
\left\{\begin{array}{l}
y_{r}(k+d-1)=y_{m}(k+d-1) \\
y_{r}(k+d+i)=\alpha y_{r}(k+d+i-1)+(1+\alpha) \omega(k+d) \\
Y_{r}=\left[y_{r}(k+d), y_{r}(k+d+1), \cdots, y_{r}(k+N)\right]^{T}
\end{array}\right.
$$

where $\omega(k)$ denotes the expected output for $k$ time, $\alpha$ indicates the output softening coefficient, and $Y_{r}$ represents the reference trajectory vector.

For the discrete system model, Eq. (3) is based on the principle of minimum variance, and the output prediction model can be expressed as follows:

$$
Y^{*}=Y_{m}+G \Delta U
$$

where $G$ is the parameter matrix. Thus, the following equations can be obtained as follows:

$$
Y^{*}=\left[y^{*}(k+d \mid k), y^{*}(k+d+1 \mid k), \cdots, y^{*}(k+N \mid k)\right]^{T}
$$




$$
\begin{aligned}
& Y_{m}=\left[y_{m}(k+d \mid k), y_{m}(k+d+1 \mid k), \cdots, y_{m}(k+N \mid k)\right]^{T} \\
& \Delta U=[\Delta u(k), \Delta u(k+1), \cdots, \Delta u(k+N-d)]^{T} \\
& \Delta u(k+i)=u(k+i)-u(k-1)
\end{aligned}
$$

where $d$ denotes the pure delay constant, and $y_{m}(t+k)$ is completely determined from the previous control input and output. The following formulas can be deduced as follows:

$$
\begin{aligned}
& y_{m}(k+j)=-\sum_{i=1}^{n_{a}} \sum_{j=1}^{N} a y_{m}(k+j-i)+ \\
& \sum_{i=1}^{n_{b}} \sum_{j=1}^{N} b_{i} u(k+j-d-i \mid k)+\sum_{j=1}^{N} \xi(k+j-i \mid k) \\
& u(k+i \mid k)=\left\{\begin{array}{l}
u(k-1), i \geq 0 \\
u(k+i), i<0
\end{array}\right. \\
& \xi(k+i \mid k)=\left\{\begin{array}{l}
0, i>0 \\
\xi(k+i), i \leq 0
\end{array}\right. \\
& y_{m}(k+i)=y(k+i), i \leq 0
\end{aligned}
$$

The performance objective function is expressed as follows:

$$
J=E\left\{\left(Y-Y_{r}\right)^{T}\left(Y-Y_{r}\right)+\Delta U^{T} \Gamma \Delta U\right\}
$$

When parameter uncertainty occurs in the system, the forgetting factor least square algorithm presented in Section 3.2 is employed to estimate the parameters. The structure is illustrated in Fig. 3.

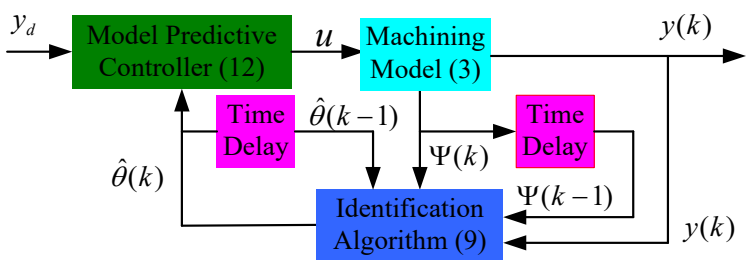

Fig. 3. Model predictive controller based on the forgetting factor least square algorithm

\section{Result analyses and dscussion}

To verify the effectiveness of the identification and control methods, the following is based on the MATLAB simulation platform.

The nominal parameters of the milling cutter servo system are as follows: the moment of inertia of the DC motor rotor is $J_{n}=8 \times 10^{-3} \mathrm{~kg} \cdot \mathrm{m}^{2}$, the friction coefficient is $B_{n}$ $=0.2 \mathrm{~N} \cdot \mathrm{m} / \mathrm{s}$, the motor resistance is $2.5 \Omega$, the inductance is $5 \mathrm{mH}$, the back electromotive force constant is $0.1 \mathrm{~V} \cdot \mathrm{s} / \mathrm{rad}$, and the torque coefficient is $10 \mathrm{~N} \cdot \mathrm{m} / \mathrm{A}$. The milling tool servo system model can be expressed as follows:

$$
G(s)=\frac{125}{s^{2}+25 s+125}
$$

When the sampling period of the system is $T_{s}=1 \mathrm{~ms}$, Eq. (22) is discretized, and the influence of external disturbance is not considered in parameter identification. The discrete model can be obtained as follows:

$$
\begin{aligned}
y(k)= & 2 y(k-1)+1 y(k-2)+ \\
& 6.1 \times 10^{-5} u(k-1)+6.1 \times 10^{-5} u(k-2)
\end{aligned}
$$

\subsection{System model parameter identification}

By considering the influence of measurement noise and other factors during identification, Eq. (23) can be expressed as:

$$
y(k)=\phi^{T} \theta+v(k)
$$

where $\quad \psi^{T}(k)=\left[\begin{array}{llll}-y(k-1) & -y(k-2) & u(k-1) & u(k-2)\end{array}\right]^{T}$ is the innovation vector. $\theta(k)=\left[\begin{array}{llll}a_{1}(k) & a_{2}(k) & b_{1}(k) & b_{2}(k)\end{array}\right]^{T}$ is the parameter vector, and its real value is $\theta_{d}=\left[\begin{array}{llll}-1.9753 & 0.9753 & 6.1982 \times 10^{-5} & 6.1468 \times 10^{-5}\end{array}\right]^{T}$. $v(k)$ applies zero mean, and variance is uniformly distributed white noise $\sigma=0.5$.

The SG method and the forgetting factor least square method are employed to identify system parameters. The identification results are presented in Fig. 4.

The following conclusions can be drawn from Fig. 4. (1) The least square identification method based on the forgetting factor achieves higher estimation accuracy than the SG method. Parameter $a_{1}$ reaches -1.980 , and its estimation error is only 0.01 . The error of the SG algorithm is 0.3 . (2) The least square identification method based on the forgetting factor exhibits faster convergence speed than the random gradient identification method. The former reaches the specified parameter value at $k=350$, while random gradient identification requires $750 \mathrm{~ms}$.

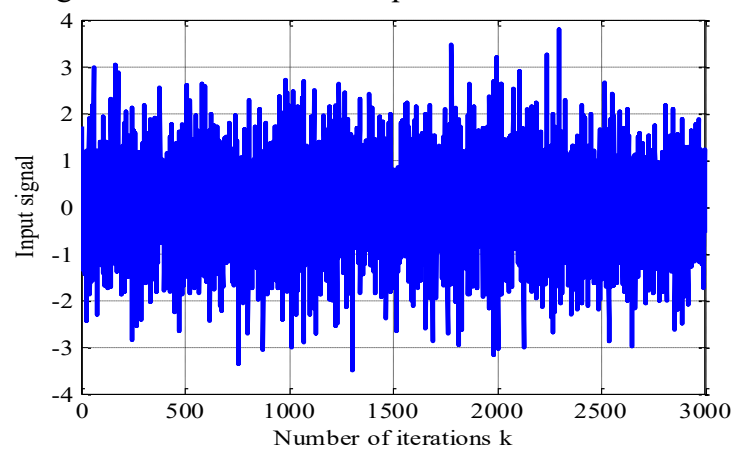

(a) Parameter estimation input signal

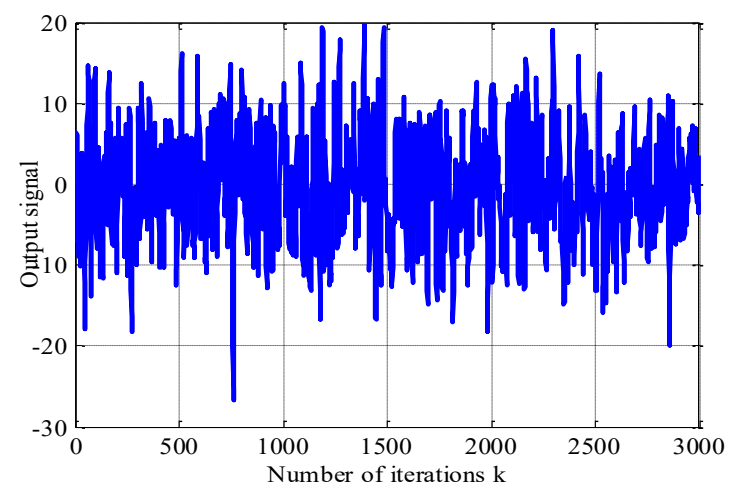

(b) Parameter estimation output signal 


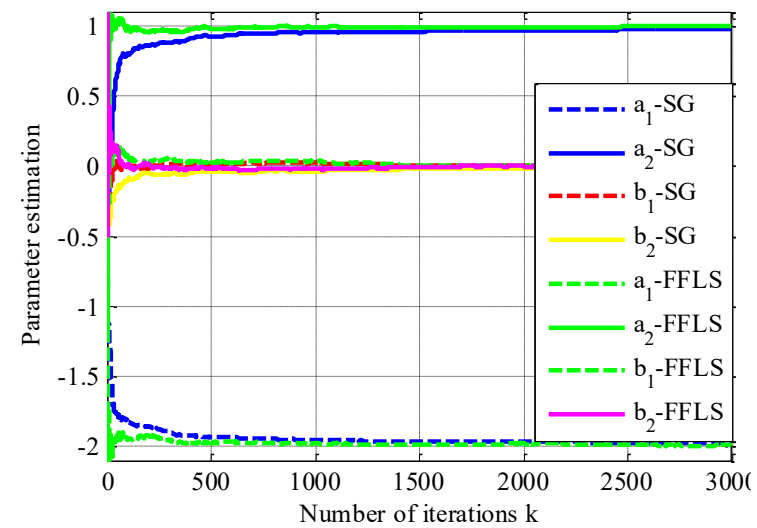

(c) Parameter estimation curve

Fig. 4. Input and output signals and parameter estimation curve in the milling process

\subsection{Control performance analysis without model parameter uncertainty}

The rotor position command signal is a step command, i.e., in a speed cycle $k \in[0300]$. When $0 \leq k \leq 150, y_{r}=10 \mathrm{rad} / \mathrm{s}$. When $150 \leq k \leq 300, y_{r}=-10 \mathrm{rad} / \mathrm{s}$. The simulation results are presented in Fig. 5.
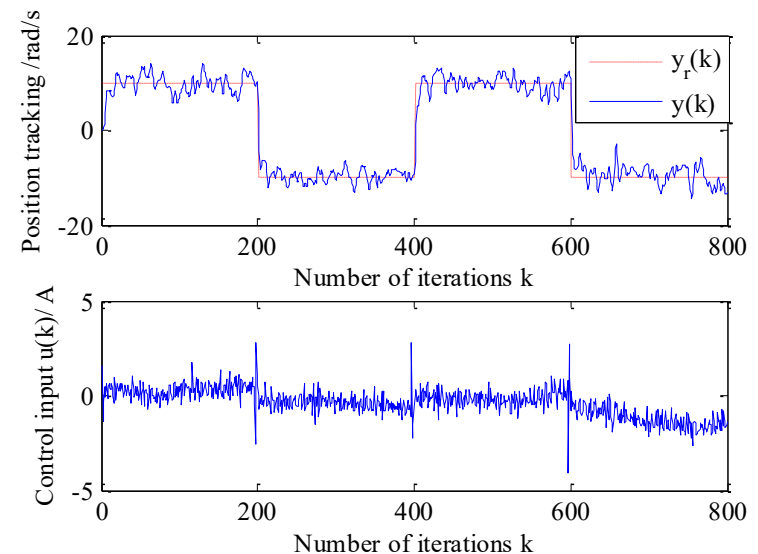

(a) Minimum variance predictive control tool speed tracking and control input curve
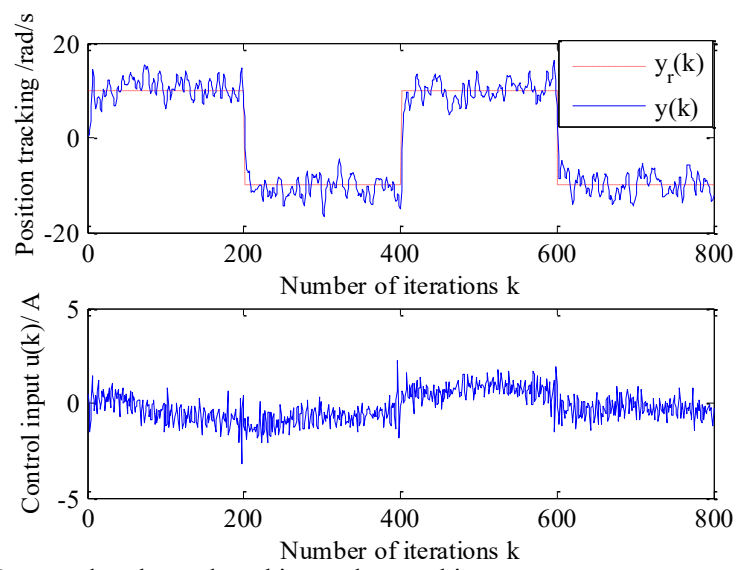

(b) Proposed tool speed tracking and control input curve

Fig. 5. Milling tool speed tracking and control input curve without model parameter uncertainty

As shown in Fig. 5, when the system exhibits no model parameter uncertainty, the traditional minimum variance predictive control and the proposed adaptive model predictive control methods can realize tool speed tracking within $10 \mathrm{~ms}(k=10)$. However, the traditional minimum variance predictive control method has a large fluctuation current when speed changes suddenly.

4.3 Controller performance analysis with model parameter uncertainty

The simulation conditions are the same as those without model parameter uncertainty. The tool speed tracking and control input curves during the milling process are shown in Fig. 6, and Fig.7 presents the least square parameter estimation curve based on the forgetting factor.
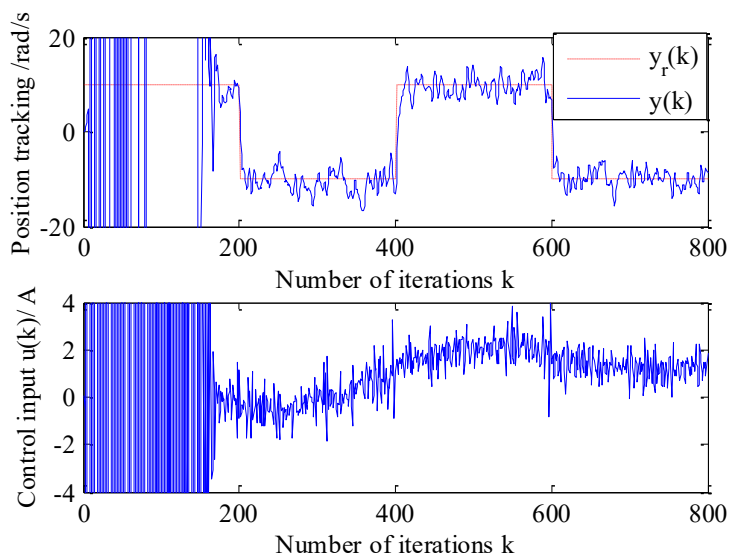

(a) Minimum variance predictive control tool speed tracking and control input curve
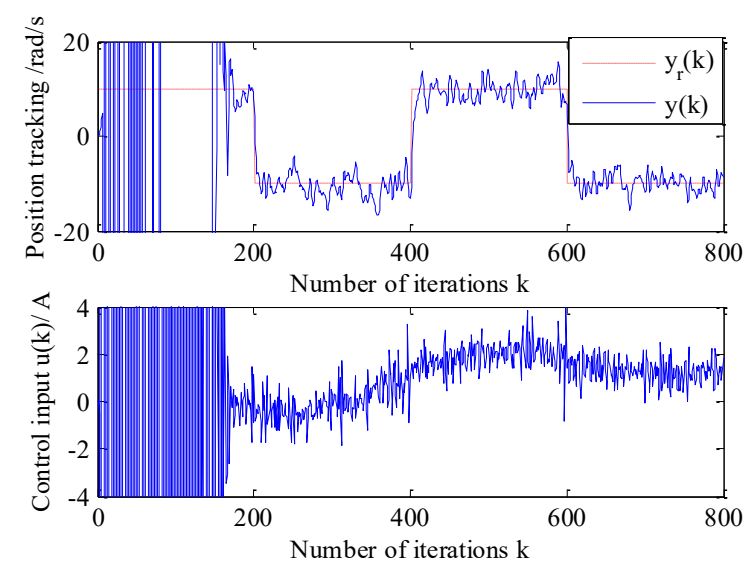

(b) Proposed tool speed tracking and control input curve

Fig. 6. Milling tool speed tracking and control input curve with model parameter uncertainty

Fig. 6 shows that when the model parameters are uncertain in the system, the following conclusions can be drawn. (1) The traditional minimum variance predictive control results in a large speed tracking error during the initial stage, reaching a saturation value of $20 \mathrm{rad} / \mathrm{s}$ and indicating that the method is completely invalid during the actual stage. By contrast, the proposed adaptive predictive control method can still accurately track speed, and only the tracking error at the starting time is relatively large. (2) The proposed adaptive predictive control method exhibits faster dynamic response capability, realizing speed tracking at $k=$ 35 (i.e., $35 \mathrm{~ms}$ ) and no overshoot occurs during the entire tracking process. Meanwhile, the traditional minimum variance predictive control requires $200 \mathrm{~ms}$ to realize speed tracking, and the tracking process fluctuates considerably, reaching $8 \mathrm{rad} / \mathrm{s}$. (3) The adaptive predictive control has smaller control input current fluctuation than the traditional minimum variance predictive control, and the control input current fluctuation is $0.5 \mathrm{~A}$. Meanwhile, the traditional minimum variance control input current fluctuation reaches 
2 A, and thus, the proposed method exhibits better dynamic performance.

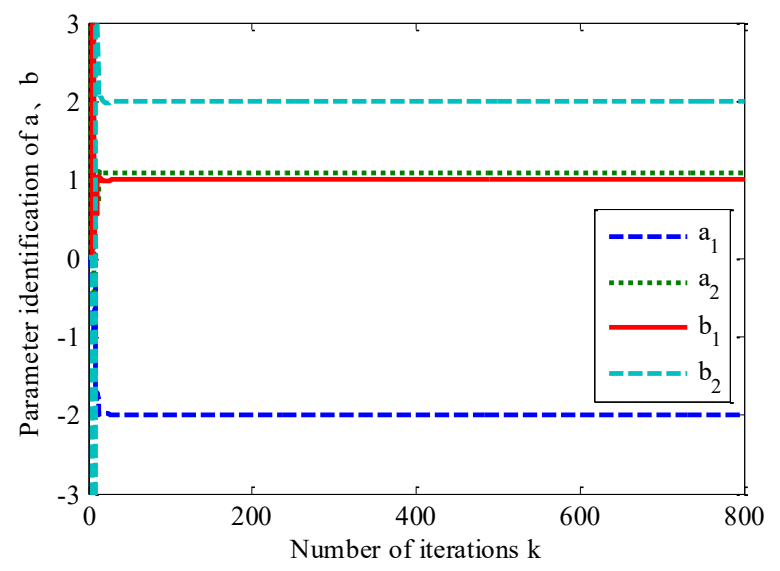

Fig. 7. Parameter online estimation curve.

Fig. 7 shows that the proposed adaptive predictive control method based on the forgetting factor least square identification algorithm can quickly realize the identification of model parameters and can cooperate with the adaptive predictive control to realize the fast tracking of tool parameters in an uncertain milling process, and the proposed method is convenient for practical engineering application.

\section{Conclusions}

To effectively improve the influences of milling process model inaccuracy and parameter uncertainty on control system accuracy, this study started from a milling process dynamic model based on system identification and model predictive control theories. Then, a new tool speed servo tracking method for the milling process was designed by combining theoretical derivation and numerical simulation. The following conclusions could be drawn as follows:

(1) Under the influence of random noise in a system, the least square identification method based on the forgetting factor achieves higher identification accuracy and speed. The accuracy of the least square identification method based on the forgetting factor is 30 times higher than that of the random gradient. The error of the former is $0.01 \mathrm{rad} / \mathrm{s}$ in $3 \mathrm{~s}$, and that of the latter reaches as high as $0.3 \mathrm{rad} / \mathrm{s}$. Identification speed is increased 2.1 times.

(2) When model parameter uncertainty does not occur in the system, the proposed adaptive model predictive control method has smaller control input current fluctuation when tool speed changes suddenly.

(3) When the parameters of the system model are uncertain, the traditional minimum variance control fails during the initial stage and it requires $200 \mathrm{~s}$ to track tool speed. By contrast, the proposed method can still track tool speed accurately and rapidly in $35 \mathrm{~s}$.

(4) When the system model parameters are uncertain, the proposed method exhibits a small control input current fluctuation, which is $1 / 4$ that of the traditional minimum variance predictive control.

On the basis of the structure model of a tool servo feed system during the milling process, a tool speed tracking method is proposed by combining theory with numerical simulation. The proposed method can improve the timevarying model parameters of the system and meet the requirements of high speed and high precision for the servo system of CNC machine tools, and thus, this method has certain application value. Given the limited experimental conditions, the proposed control method should be further verified and optimized through subsequent experiments. In particular, the proposed method disregards the influence of external disturbance on the tool feed servo system for the milling process during identification and control. A study under the influence of this factor will be more accurate in accordance with the experimental platform.

\section{Acknowledgements}

This study was supported by the Key Scientific Study Projects of the Higher Education Institutions of Henan Province (Grant Nos. 20B470003 and 18B470007) and the Promotion Special Project of the Scientific Study Program of Henan Province (Grant Nos. 202102210084 and 202102210298).

This is an Open Access article distributed under the terms of the Creative Commons Attribution License

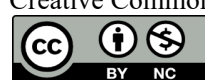

\section{References}

1. Liu, X.L., Su, C.Y., Yang, F., "FNN approximation based active dynamic surface control for suppressing chatter in micro-milling with piezo-actuators". IEEE Transactions on Systems, Man and Cybernetics Systems, 47(8), 2017, pp.2100-2113.

2. Zhang, C.Y., Jiang, P.Y., "RFID-driven energy-efficient control approach of CNC machine tools using deep belief networks". IEEE Transactions on Automation Science and Engineering, 17(1), 2020, pp.129-141.

3. Sun, D., Tong, M.C., "A synchronization approach for the minimization of contouring errors of CNC machine tools". IEEE Transactions on Automation Ence and Engineering, 6(4), 2009, pp.720-729.

4. Aslan, D., Altintas, Y., "Prediction of cutting forces in five-axis milling using feed drive current measurements". IEEE/ASME Transactions on Mechatronics, 23(2), 2018, pp.833-844.

5. Chen, C.S., Sun, Y.T.A., "Intelligent computer-aided processing planning of multi-axis CNC tapping machine". IEEE Access, (5), 2017, pp.2913-2920.

6. Zhu, K.P., Zhang, Y., "A cyber-physical production system framework of smart CNC machining monitoring system". IEEE/ASME Transactions on Mechatronics, 23(6), 2018, pp.25792586.
7. Wang, P., Wang, H., Chen, X., "Research on reliability comprehensive evaluation method of five-axis $\mathrm{CNC}$ machine tools based on AHP and extension theory". The Journal of Engineering, (23), 2019, pp.8599-8603.

8. Odhano, S.A., Pescetto, P., Awan, H.A.A., Hinkkanen, M., Pellegrino, G., Bojoi, R., "Parameter identification and selfcommissioning in AC motor drives: a technology status review". IEEE Transactions on Power Electronics, 34(4), 2019, pp.36033614.

9. Xu, P., Xiao, J., Li, S., Peng, X.F., "Forgetting factor based stochastic gradient identification for permanent magnet synchronous motor". Small and Special Electrical Machines, 42(4), 2014, pp.1-3, 7.

10. Qu, B., Sun, X.F., Zhang, X.H., Huang, W., Su, J., Du, S.H., Zhai, Z.Q., Sun, R.N., Lou, Z.Y., "Correction model of energy consumption evaluation of cluster motor system based on levenberg - marquardt algorithm". Transactions of the Chinese Society of Agricultural Engineering, 34(18), 2018, pp.44-50.

11. Accetta, A., Cirrincione, M., Pucci, M., Sferlazza, A., "State space vector model of linear induction motors including end-effects and iron losses-Part II: model identification and results". IEEE Transactions on Industry Applications, 56(1), 2020, pp.245-255. 
12. Zhang, L.W., Zhang, P., Liu, Y.F., Zhang, C., Liu, J., "Parameter identification of permanent magnet synchronous motor based on variable step-size Adaline neural network". Transactions of China Electrotechnical Society, 33(S2), 2018, pp.377-384.

13. Fagiano, L., Lauricella, M., Angelosante, D., Ragaini, E., "Identification of induction motors using smart circuit breakers". IEEE Transactions on Control Systems Technology, 27(6), 2019, pp.2638-2646.

14. Paul, S., Morales-Menendez, R., "Active control of chatter in milling process using intelligent PD/PID control". IEEE Access, (6), 2018, pp.72698-72713.

15. Angel, L., Viola, J., "Design and statistical robustness analysis of FOPID, IOPID and SIMC PID controllers applied to a motorgenerator system". IEEE Latin America Transactions, 13(12), 2015, pp.3724-3734.

16. Corapsiz, M.F., Erenturk, K., "Trajectory tracking control and contouring performance of three-dimensional CNC". IEEE Transactions on Industrial Electronics, 63(4), 2016, pp.2212-2220.

17. Hanifzadegan, M., Nagamune, R., "Contouring control of CNC machine tools based on linear parameter-varying controllers". IEEE/ASME Transactions on Mechatronics, 21(5), 2016, pp.25222530 .
18. Heydari, A., Landers, R.G., Balakrishnan, S.N., "Optimal control approach for turning process planning optimization". IEEE Transactions on Control Systems Technology, 22(4), 2014, pp.1337-1349.

19. Zhou, C.G., Guo, K., Yang, B., Wang, H., Sun, J., Lu, L., "Singularity analysis of cutting force and vibration for tool condition monitoring in milling". IEEE Access, (7), 2019, pp. 134113-134124.

20. Wu, Y., Zhang, H.T., Huang, T., Ren, G.P., Ding, H., "Robust chatter mitigation control for low radial immersion machining processes". IEEE Transactions on Automation Science and Engineering, 15(4), 2018, pp.1972-1979.

21. Huang, W.S., Liu, C.W., Hsu, P.L., Yeh, S.S., "Precision control and compensation of servomotors and machine tools via the disturbance observer". Transactions on Industrial Electronics, 57(1), 2010, pp.420-429.

22. Di, C.S., Goldsmith, A., Kalabic, U.V., Bortoff, S.A., "Cascaded reference governor-MPC for motion control of two-stage manufacturing machines". IEEE Transactions on Control Systems Technology, 27(5), 2019, pp.2030-2044.

23. Yuan, Y., Zhang, H.T., Wu, Y., Zhu, T., Ding, H., "Bayesian learning-based model-predictive vibration control for thin-walled workpiece machining processes". IEEE/ASME Transactions on Mechatronics, 22(1), 2017, pp.509-520. 